\title{
Solution of a Birkhoff Interpolation Problem by a Special Spline Function
}

\author{
Ambrish Kumar Pandey \\ Department of Mathematics \\ Integral University \\ Lucknow-226026
}

(INDIA)

\author{
K. B. Singh \\ Associate Professor \\ Dept. of Maths. \& Comp. Sc. \\ The Papua New Guinea \\ University of Technology \\ LAE, Papua New Guinea
}

\author{
Qazi Shoeb Ahmad \\ Associate Professor \\ Department of Mathematics \\ Integral University \\ Lucknow-226026
}

(INDIA) 


$$
s_{\Delta}(x)=
$$

$f_{0}+\left(x-x_{0}\right) f_{0}^{\sigma}+\frac{\left(x-x_{0}\right)^{2}}{2 !} f_{0}^{w}+\frac{\left(x-x_{0}\right)^{g}}{3 !} a_{0,3}+$ $\frac{\left(x-x_{0}\right)^{4}}{4 !} a_{0,4}+\frac{\left(x-x_{0}\right)^{5}}{5 !} a_{0,5}$

$$
s_{k}(x)=
$$

$f_{k}+\left(x-x_{k}\right) f_{k}^{r}+\frac{\left(x-x_{k}\right)^{2}}{2 !} a_{k, 2}+$

$\frac{\left(x-x_{k}\right)^{n}}{3 !} a_{k, 3}+\frac{\left(x-x_{k}\right)^{4}}{4 !} a_{k, 4}+\frac{\left(x-x_{k}\right)^{5}}{5 !} a_{k, 5}$

For determining the coefficients we apply the interpolatory condition (1.1) and the continuity requirements that

$s_{\Delta}\left(x_{k}\right) \in C^{2}(I)$. Then we have

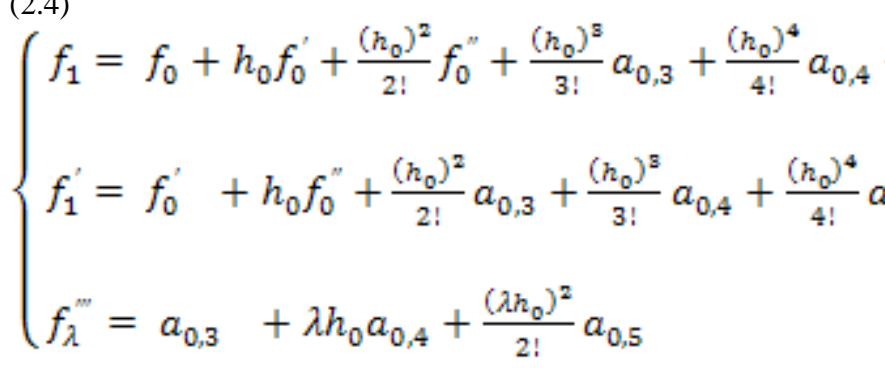

$$
\left\{\begin{array}{l}
f_{k+1}=f_{k}+h_{k} f_{k}^{\sigma}+\frac{\left(h_{k}\right)^{2}}{2 !} a_{k, 2}+\frac{\left(h_{0}\right)^{\mathrm{s}}}{3 !} a_{k, 3}+\frac{\left(h_{0}\right)^{4}}{4 !} \\
f_{k+1}^{\sigma}=f_{k}^{\sigma}+h_{k} a_{k, 2}+\frac{\left(h_{k}\right)^{2}}{2 !} a_{k, 3}+\frac{\left(h_{0}\right)^{3}}{3 !} a_{k, 4}+\frac{(}{f_{k+\lambda}^{m}=a_{k, 3}+\lambda h_{k} a_{k, 4}+\frac{\left(\lambda h_{0}\right)^{2}}{2 !} a_{k, 5},}
\end{array}\right.
$$$$
\mathrm{k}=1,2
$$

and (2.6)

$$
\left\{\begin{array}{l}
a_{k+1}=a_{k}+h_{k} a_{k, 3}+\frac{\left(h_{k}\right)^{2}}{2 !} a_{k_{s}, 4}+\frac{\left(h_{k}\right)^{s}}{3 !} a_{k_{z} 5} \\
a_{1,2}=f_{0}^{w}+h_{0} a_{0,3}+\frac{\left(h_{0}\right)^{2}}{2 !} a_{0,4}+\frac{\left(h_{0}\right)^{s}}{3 !} a_{0,5}
\end{array}\right.
$$

(2.7)

$a_{0,5}=\frac{1}{\left(10 \lambda^{2}-8 \lambda+1\right)}\left[\frac{480(3 \lambda-1)}{h_{0}^{5}}\left(f_{1}-f_{0}-\right.\right.$ $\left.h_{0} f_{0}^{\sigma}-\frac{h_{0}^{x}}{2 !} f_{0}^{*}\right)-\frac{120(4 \lambda-1)}{h_{0}^{4}}\left(f_{1}^{\sigma}-f_{0}^{\sigma}-h_{0} f_{0}^{\sigma}\right)+$ $\left.\frac{20 f_{\lambda}^{m}}{h_{0}^{2}}\right]$

$a_{0,4}=\frac{1}{\left(10 \lambda^{2}-8 \lambda+1\right)}\left[\frac{-120\left(6 \lambda^{2} 1\right)}{h_{0}^{4}}\left(f_{1}-f_{0}-\right.\right.$

$\left.h_{0} f_{0}^{\sigma}-\frac{h_{0}^{2}}{2 !} f_{0}^{\sigma}\right)+\frac{24\left(10 \lambda^{2}-1\right)}{h_{0}^{s}}\left(f_{1}^{\sigma}-f_{0}^{\sigma}-h_{0} f_{0}^{\sigma}\right)-$ $\left.\frac{8 f_{\lambda}}{h_{\mathrm{p}_{2}}}\right]$

(2.9)

$a_{0,3}=\frac{1}{\left(10 \lambda^{2}-8 \lambda+1\right)}\left[\frac{120(2 \lambda-1)}{h_{0}^{\mathrm{a}}}\left(f_{1}-f_{0}-\right.\right.$ $\left.h_{0} f_{0}^{\sigma}-\frac{h_{0}^{2}}{2 x} f_{0}^{\infty}\right)-\frac{12(5 \lambda-2)}{h_{0}^{2}}\left(f_{1}^{\sigma}-f_{0}^{\sigma}-h_{0} f_{0}^{\sigma}\right)+$ $\left.f_{\lambda}^{m}\right]$ From (2.5) we have,

$a_{k_{s} 5}=\frac{1}{\left(30 \lambda^{2}-24 \lambda+3\right)}\left[\frac{1440(3 \lambda-1)}{h_{k}^{5}}\left(f_{k+1}-f_{k}-\right.\right.$ $\left.h_{k} f_{k}^{\mathrm{g}}\right)-\frac{20(72 \lambda-18)}{h_{k}^{4}}\left(f_{k+1}^{v}-f_{k}\right)+$ $\frac{20(18-36 \lambda)}{h_{k}^{\mathrm{s}}} a_{k_{2} 2}+$ $\left.+\frac{60}{h_{k}^{2}} f_{k+\lambda}^{w}\right]$

$a_{k, 4}=\frac{1}{\left(30 \lambda^{2}-24 \lambda+3\right)}\left[\frac{\left(-2160 \lambda^{2}+360\right)}{h_{k}^{4}}\left(f_{k+1}-\right.\right.$ $\left.f_{k}-h_{k} f_{k}^{\sigma}\right)+\frac{\left(720 \lambda^{2}-72\right)}{h_{k}^{\mathrm{s}}}\left(f_{k+1}^{8}-f_{k}^{\mathrm{g}}\right)+$ $+\frac{\left(360 \lambda^{2}-108\right)}{h_{k}^{2}} a_{k_{2} 2}-$ $\left.-\frac{24}{h_{k}} f_{k+\lambda}^{\mathrm{w}}\right]$ 
(2.12)

$$
\begin{gathered}
a_{k, 3}=\frac{1}{\left(30 \lambda^{2}-24 \lambda+3\right)}\left[\frac { ( 7 2 0 \lambda ^ { 2 } - 3 6 0 \lambda ) } { h _ { k } ^ { s } } \left(f_{k+1}-\right.\right. \\
\left.f_{k}-h_{k} f_{k}^{5}\right)+\frac{\left(72 \lambda-180 \lambda^{2}\right)}{h_{k}^{2}}\left(f_{k+1}^{v}-f_{k}\right)+ \\
\left.\begin{array}{cc}
\frac{\left(108 \lambda-180 \lambda^{2}\right)}{h_{k}} a_{k, 2}+ & \left.+3 f_{k+\lambda}^{\mathrm{m}}\right]
\end{array}\right]
\end{gathered}
$$

Using values of these coefficients in (2.6) we get

(2.13)

$$
\begin{aligned}
& a_{1,2}= \\
& \frac{1}{\left(10 \lambda^{2}-8 \lambda+1\right)}\left[\frac{\left(20 \lambda^{3}-34 \lambda^{2}+18 \lambda-3\right)}{(2 \lambda-1)} f_{0}^{\omega}+\frac{1}{3} h_{0} f_{\lambda}^{m}+\right. \\
& \frac{20\left(-6 \lambda^{2}+6 \lambda-1\right)}{h_{0}^{2}}\left(f_{1}-f_{0}-h_{0} f_{0}\right)+ \\
& +\frac{\left(120 \lambda^{\mathrm{I}}-172 \lambda^{2}+72 \lambda-\right.}{(2 \lambda-1) h_{0}} \\
& \left.\left.f_{0}\right)\right] \\
& \text { (2.14) } a_{k+1,2} \\
& +\frac{\left(-30 \lambda^{2}+36 \lambda-9\right)}{\left(30 \lambda^{2}-24 \lambda+3\right)} a_{k_{3} 2}= \\
& \frac{1}{\left(30 \lambda^{2}-24 \lambda+3\right)}\left[\begin{array}{l}
\frac{\left(-360 \lambda^{2}-360 \lambda-60\right)}{h_{k}^{2}}\left(f_{k+1}-f_{k}-h_{k} f_{k}^{v}\right) \\
+\frac{\left(180 \lambda^{2}-168 \lambda+24\right)}{h_{k}}\left(f_{k+1}^{*}-f_{k}^{\sigma}\right)+h_{k} f_{k}^{\pi}
\end{array}\right.
\end{aligned}
$$

The coefficient matrix of the system of equations (2.13) and (2.14) in the unknowns $a_{k, 2}, \mathrm{k}=1,2, \ldots, \mathrm{n}-1$ is seen to be nonsingular and hence the coefficients $a_{k, 2}, \mathrm{k}=1,2, \ldots, \mathrm{n}$ 1 , are uniquely determined and so are, therefore, the coefficients $a_{k_{2} 3}, a_{k_{2} 4}, a_{k_{z} 5}, \mathrm{k}=1,2, \ldots, \mathrm{n}-1$.

\section{THEOREM OF CONVERGENCE}

Let $f \in C^{l}(I), l=5,6$. Then for the unique spline $\mathrm{s}_{\Delta}(\mathrm{x})$ of Theorem 1 associated with the function $f$, we have

(3.1)

$$
\begin{aligned}
& \| s_{\Delta}^{(5)}(x)- \\
& f^{(5)}(x) \| \begin{cases}O\left(\omega_{5}(H)\right), & \text { if } f \in \\
K_{3} H\left\|f^{(6)}\right\|+o\left(\omega_{5}(H)\right), & \text { if } f \in\end{cases}
\end{aligned}
$$

And if $\frac{\max h_{k}}{\min h_{k}} \leq \lambda \leq \infty$ and $\mathrm{H}=$

$\max _{0 \leq k \leq n-1} h_{k}$, then

(3.2)

$$
\begin{aligned}
& \| s_{\Delta}^{(q)}(x)- \\
& f^{(q)}(x) \|\left\{\begin{array}{c}
o\left(H^{4-q} \omega_{5}(H)\right), \\
K_{2} H^{6-q}\left\|f^{(6)}\right\|+o\left(H^{5-q} \omega_{5}(H)\right), \text { if } f \in C^{6}(I),
\end{array}\right. \\
& q=0,1,2,3,4 . \\
& \text { Where } K_{2} \text { and } K_{3} \text { are some constants involving } \lambda \\
& (0 \leq \lambda \leq 1) .
\end{aligned}
$$

\subsection{Auxiliary Lemmas}

Now we give three lemmas that are used to obtain the proof of the Theorem of convergence theorem.

\subsubsection{Lemma}

$$
\text { Let } \quad A_{k_{2} 2}=a_{k, 2}-f_{k}^{w} \text {. }
$$

Then we have for $\mathrm{k}=1,2, \ldots, \mathrm{n}-1$.

$$
\begin{gathered}
\left|A_{k, 2}\right|=\left\{\begin{array}{c}
o\left(\sum_{v=0}^{k-1} h_{v}^{3} \omega_{5}\left(h_{v}\right)\right), \quad \text { if } f \in C^{5}(I) \\
K_{1} h_{k}^{4} f^{(6)}+o\left(\sum_{v=0}^{k-1} h_{v}^{4} \omega_{6}\left(h_{v}\right)\right), \text { if } f \in C^{6}(I)
\end{array}\right. \\
\text { Where } \mathrm{K}_{1}=\frac{\left(20 \lambda^{\mathrm{s}}-30 \lambda^{2}+12 \lambda-1\right)}{120\left(30 \lambda^{2}-24 \lambda+3\right)} .
\end{gathered}
$$

Proof From (2.14) we have

$$
\begin{aligned}
& \text { (3.1.1) } A_{k+1,2}+\frac{\left(-30 \lambda^{2}+36 \lambda-9\right)}{\left(30 \lambda^{2}-24 \lambda+3\right)} A_{k, 2}= \\
& \begin{array}{r}
\left(a_{k+1,2}-f_{k+1}\right)+\frac{\left(-30 \lambda^{2}+36 \lambda-9\right)}{\left(30 \lambda^{2}-24 \lambda+3\right)}\left(a_{k, 2}-f_{k}^{\omega}\right) \\
=\alpha_{k} \text { (say), } \mathrm{k}=1,2,
\end{array}
\end{aligned}
$$

$\ldots \ldots, \mathrm{n}-2$

$$
\begin{aligned}
& \alpha_{k}= \\
& \frac{1}{\left(30 \lambda^{2}-24 \lambda+3\right)}\left[\frac{\left(-360 \lambda^{2}+360 \lambda-60\right)}{h_{k}^{2}}\left(f_{k+1}-f_{k}-h_{k} f_{k}^{\prime}\right)+\frac{\left(180 \lambda^{2}-168 \lambda+24\right)}{h_{k}}\left(f_{k+1}^{\prime}-f_{k}^{\prime}\right)+h_{k} f_{k+\lambda}^{\prime \prime \prime}\right]- \\
& \quad-\left[f_{k+1}^{\prime \prime}+\frac{\left(-30 \lambda^{2}+36 \lambda-9\right)}{\left(30 \lambda^{2}-24 \lambda+3\right)} f_{k}^{\prime \prime}\right]
\end{aligned}
$$

If $f \in C^{5}(I)$ then by Taylor's formula$$
\alpha_{k}=O\left(h_{k}^{3} \omega_{5}\left(h_{k}\right)\right) \text {. }
$$

Similarly if if $f \in C^{5}(I)$, then

$\alpha_{k}=K_{1} h_{k}^{4} f_{k}^{(6)}+o\left(h_{k}^{4} \omega_{6}\left(h_{k}\right)\right)$. 
(3.1.4) $\left|A_{1,2}\right|=$

$\left|a_{1,2}-f_{1}^{\prime \prime}\right|=$

$\left\{\begin{array}{r}o\left(h_{0}^{3} \omega_{5}\left(h_{v}\right)\right) \text {, if } f \in C^{5}(1 \\ K_{2} h_{0}^{4} f^{(6)}+O\left(h_{0}^{4} \omega_{6}\left(h_{v}\right)\right), \text { if } f \in C^{6}(I) \text { Where }\end{array}\right.$

From (3.1.1) and (3.1.2) and the derivatives for $\alpha_{k}$ we have

$\left|A_{k, 2}\right|=$

$\left\{\begin{array}{c}o\left(\sum_{v=0}^{k-1} h_{v}^{3} \omega_{5}\left(h_{v}\right)\right), \text { if } f \in C^{5}(I) \\ K_{1} h_{k}^{4} f^{(6)}+O\left(h_{k}^{4} \omega_{6}\left(h_{k}\right)\right), \text { if } f \in C^{6}(I)\end{array}\right.$

This proves the assertion of lemma.

\subsubsection{Lemma}

Let $\quad A_{k_{3} 4}=a_{k_{s} 4}-f_{k}^{(4)} \quad$ and $\frac{\max h_{k}}{\min h_{k}} \leq$

$\lambda \leq \infty, \quad \mathrm{H}=\max _{0 \leq k \leq n-1} h_{k}$.

Then we have for $\mathrm{k}=0,1, \ldots \ldots, \mathrm{n}-1$.

$$
\begin{aligned}
& \left|A_{k_{2}, 4}\right|= \\
& O\left(\omega_{5}(H)\right), \text { if } f \in C^{5}(I) \\
& K_{2} H^{2}\left\|f^{(6)}\right\|+O\left(H \omega_{6}(H)\right), \text { if } f \in C^{6}(I)
\end{aligned}
$$

Where

$\mathrm{K}_{2}=$

$\frac{-40 \lambda^{3}+30 \lambda^{2}-1}{10\left(30 \lambda^{2}-24 \lambda+3\right)}$

Proof From (2.8) and (2.11) we see $A_{0,2}=0$, then

(3.1.5) $A_{k, 4}=a_{k, 4}-f_{k}^{(4)}=\frac{\left(360 \lambda^{2}-108\right)}{\left(30 \lambda^{2}-24 \lambda+3\right) h_{k}^{2}}$

$A_{k, 2}+\beta_{k}, \quad \mathrm{k}=0,1, \ldots \ldots, \mathrm{n}-1$

Where $\beta_{k}=$

$\frac{1}{\left(30 \lambda^{2}-24 \lambda+3\right)}\left[\frac{\left(-2160 \lambda^{2}+360\right)}{h_{k}^{4}}\left(f_{k+1}-f_{k}-h_{k} f_{k}\right)+(\right.$

$$
\beta_{k}=O\left(h_{k} \omega_{5}(H)\right) \text {, if } f \in C^{5}(I) .
$$

If $\mathrm{f} \in \mathrm{C}^{6}(\mathrm{I})$, then

$\beta_{k}=K_{2} h_{k}^{2} f_{k}^{(6)}+O\left(h_{k}^{2} \omega_{6}\left(h_{k}\right)\right)$, where $K_{2}$

$=\frac{\left(-40 \lambda^{\mathrm{g}}+30 \lambda^{2}-1\right)}{10\left(30 \lambda^{2}-24 \lambda+3\right)}$.
Using Lemma 3.1, we have for $\mathrm{k}=0,1$ n-1.

$\left|A_{k, 4}\right|=\left\{\begin{array}{l}O\left(\frac{1}{h_{k}^{2}} \sum_{v=0}^{k-1} h_{v}^{3} \omega_{5}\left(h_{v}\right)\right)+O\left(h_{k} \omega_{5}\left(h_{k}\right)\right), \quad \text { if } f \in C^{5}(I) \\ K_{2} h_{k}^{2} f_{k}^{(6)}+O\left(h_{k}^{2} \omega_{6}\left(h_{k}\right)\right), \text { if } f \in C^{6}(I)\end{array}\right.$

The result clearly holds for $\mathrm{k}=0$. Hence if $\frac{\max h_{k}}{\min h_{k}} \leq \lambda$

$\leq \infty, \quad \mathrm{H}=\max _{0 \leq k \leq n-1} h_{k}$,

we have from (3.1.5) to (3.1.7)

$\left|A_{k_{4}}\right|=$

$\left\{\begin{array}{c}O\left(\omega_{5}(H)\right), \text { if } f \in C^{5}(I) \\ K_{2} H\left\|f^{(6)}\right\|+O\left(H \omega_{6}(H)\right), \text { if } f \in C^{6}(I) \\ \mathrm{k}=0,1, \ldots \ldots, \mathrm{n}-1 .\end{array}\right.$

This proves Lemma 3.1.2.

\subsubsection{Lemma}

$$
\text { Let } A_{k_{s} 5}=a_{k_{s} 5}-f_{k}^{(5)}
$$

Then we have for $\mathrm{k}=0,1, \ldots ., \mathrm{n}-1$

$$
\begin{aligned}
& \left|A_{k_{3}, 5}\right|= \\
& \left\{\begin{array}{l}
o\left(\omega_{5}(H)\right), \text { if } f \in C^{5}(I) \\
K_{3} H\left\|f^{(6)}\right\|+O\left(H \omega_{6}(H)\right), \text { if } f \in C^{6}(I)
\end{array}\right. \\
& \text { Where } \mathrm{K}_{2}=\frac{\left(10 \lambda^{3}-6 \lambda+1\right)}{10\left(30 \lambda^{2}-24 \lambda+3\right)} .
\end{aligned}
$$

Proof Following similar method we can get the results for $\left|A_{k_{2} 5}\right|$ hence we omitted the proof.

\section{PROOF OF THEOREM 2}

Let $x \in\left[x_{k}, x_{k+1}\right], \mathbf{k}=\mathbf{0}, \mathbf{1}, \ldots . ., \mathbf{n}-\mathbf{1}$

Then from (2.3) we have

(4.1) $s_{k}^{(5)}(x)$

$=a_{k, 5}$

(4.2) $s_{k}^{(5)}(x)$

$=a_{k_{j} 4}+\left(x-x_{k}\right) a_{k_{s} 5}$

Therefore

$\left|s_{k}^{(5)}(x)-f^{(5)}(x)\right|$
$=\left|s_{k}^{(5)}(x)-f_{k}^{(5)}+f_{k}^{(5)}-f^{(5)}(x)\right|$ 


$$
\begin{aligned}
& \leq\left|a_{k, 5}-f_{k}^{(5)}\right|+ \\
& \left|f_{k}^{(5)}-f^{(5)}(x)\right| .
\end{aligned}
$$

If $\mathrm{f} \in \mathrm{C}^{5}$ (I) then using Lemma 3.1.3, we have

$$
\left|s_{k}^{(5)}(x)-f^{(5)}(x)\right|=O\left(\omega_{5}(H)\right)^{(4.3)}
$$

Again from (4.2)

$$
\begin{aligned}
& (4.4) s_{k}^{(4)}(x)-f^{(4)}(x)=\left(a_{k, 4}-f^{(4)}\right)+ \\
& \left(x-x_{k}\right)\left(a_{k, 5}-f_{k}^{(5)}\right)-[ \\
& \left.f^{(4)}(x)-f_{k}^{(4)}-\left(x-x_{k}\right) f_{k}^{(5)}\right]
\end{aligned}
$$$$
=A_{k_{4} 4}
$$

$+\left(x-x_{k}\right) A_{k_{j}, 5}-\left(x-x_{k}\right)\left(f^{(4)}\left(\eta_{k}\right)-f_{k}^{(5)}\right)$, $x_{k} \leq \eta_{k} \leq x$

Thus,

$\left|s_{k}^{(5)}(x)-f^{(5)}(x)\right| \leq\left|A_{k_{4}, 4}\right|+H\left|A_{k_{,} 5}\right|+$ $H \omega_{5}(H)$

Now applying Lemma 3.1.2 and 3.1.3 we get,

$$
\begin{aligned}
& \text { (4.5) }\left|s_{k}^{(4)}(x)-f^{(4)}(x)\right|=O\left(\omega_{5}(H)\right) \\
& +H O\left(\omega_{5}(H)\right)=O\left(\omega_{5}(H)\right) . \\
& \text { Now, }\left|s_{k}^{\mathrm{w}}(x)-f^{\mathrm{m}}(x)\right|= \\
& \left|\int_{x_{k+\lambda}}^{x}\left[s_{k}^{(4)}(t)-f^{(4)}(t)\right] d t\right| \leq \\
& \left(x-x_{k+\lambda}\right)\left|s_{k}^{(4)}(x)-f^{(4)}(x)\right| \\
& \text { (4.6) }\left|s_{k}^{\mathrm{w}}(x)-f^{\mathrm{w}}(x)\right|=\left(H \omega_{5}(H)\right) . \\
& \text { Set } \mathrm{h}\left(x_{k}\right)=\mathrm{h}\left(x_{k+1}\right)=0 .
\end{aligned}
$$

So by Rolle's theorem, there exists a $\mu_{k}$,

$x_{k}<\mu_{k}<x_{k+1}$, such that $h^{\circ}\left(\mu_{k}\right)=s_{k}^{w}\left(\mu_{k}\right)$ -

$$
f^{m}\left(\mu_{k}\right)=0 \text {. }
$$

This gives $\quad\left|s_{k}^{\prime \prime}(x)-f^{\prime \prime}(x)\right|=$ $\left|\int_{\mu_{k}}^{\infty}\left[s_{k}{ }^{\mathrm{m}}(t)-f^{\mathrm{m}}(t)\right] d t\right| \leq$ $\left(x-\mu_{k}\right)\left|s_{k}^{\prime \prime \prime}(x)-f^{\prime \prime \prime}(x)\right|$

$$
=O\left(H H \omega_{5}(H)\right)
$$

$$
\left|s_{k}^{\prime \prime}(x)-f^{\prime \prime}(x)\right|=\left(H^{2} \omega_{5}(H)\right) .
$$

Again using interpolatory conditions (1.1) we can write

$$
\begin{gathered}
\left|s_{k}^{\sigma}(x)-f^{v}(x)\right|= \\
\left|\int_{x_{k}}^{x}\left[s_{k}^{w}(t)-f^{w}(t)\right] d t\right| \\
\text { (4.8) } \quad\left|s_{k}^{\sigma}(x)-f^{v}(x)\right|=\left(H^{3} \omega_{5}(H)\right) .
\end{gathered}
$$

Similarly

$$
\begin{aligned}
& \text { (4.9) }\left|s_{k}(x)-f(x)\right|= \\
& \begin{aligned}
\left|\int_{x_{k}}^{x}\left[s_{k}^{v}(t)-f^{v}(t)\right] d t\right| & \\
& =O\left(H^{4} \omega_{5}(H)\right) .
\end{aligned}
\end{aligned}
$$

This proves the theorem for $f \in C^{5}(I)$. Next we consider the case when $f \in C^{6}(I)$. Then from Lemma 3.1.3

$$
\begin{aligned}
& \left|s_{k}^{(5)}(x)-f^{(5)}(x)\right|= \\
& \left|\left(a_{k_{x} 5}-f^{(5)}\right)+\left(x-x_{k}\right) f_{k}^{(5)}\left(\xi_{k}\right)\right|, \\
& x_{k} \leq \xi_{k} \leq x \\
& o\left(H \omega_{6}(H)\right) .
\end{aligned}
$$

Again

$$
\begin{aligned}
& \quad s_{k}^{(4)}(x)-f^{(4)}(x)= \\
& A_{k_{2}, 4}+\left(x-x_{k}\right) A_{k_{x} 5}+\frac{\left(x-x_{k}\right)^{2}}{2} f^{(5)}\left(\xi_{k}\right), \\
& x_{k} \leq \xi_{k} \leq x
\end{aligned}
$$

Which on using Lemma 3.2 and Lemma 3.3, gives

(4.10) $\left|s_{k}^{(4)}(x)-f^{(4)}(x)\right| \leq K_{3} H\left\|f^{(6)}\right\|+$ $\left(H \omega_{6}(H)\right)$.

From (4.10) on using method of successive integration we at once have

(4.11) $\left|s_{k}^{(q)}(x)-f^{(q)}(x)\right| \leq K_{3} H^{6-q}\left\|f^{(6)}\right\|+$ $\left(H^{6-q} \omega_{6}(H)\right), \mathrm{q}=1,2,3,4$.

This proves the theorem of convergence for $f \in C^{6}(I)$.

\section{REFERENCES}

[1] Burkett, J. and Verma, A.K. (June-1995) On Birkhoff Interpolation $(0 ; 2)$ case, Aprox. Theory and its Appl. $11 ; 2$.

[2] Chawala, M. M., Jain, M.K. and Subramanian, R. (1990) On numerical Integration of a singular two-point boundary value problem Inter. J. Computer. Math. Vol 31, 187-194.

[3] Cheney, E. W. (1966) Interpolation to approximation Theory, McGraw Hill, New York. 
[4] Davydov, O. (1997) On almost Interpolation, Journal of Approx. Theory 91(3), 396-418.

[5] Goodman, T.N.T. ; Ivanov, K.G. ; Sharma, A.(1996) Hermite interpolation in the roots of unity, Journal of Approx. Theory, 84(1), 41-60.

[6] Jhunjhunwala, N. and Prasad, J. (1994) on some regular and singular problems of Birkhoff interpolation, Internet. J. Math. \& Math. Sci. 17 No.2, 217-226.

[7] Lorentz, G.G., (1973) Approximation Theory, Academic Press Inc. New York.

[8] Lorentz, G.G., Jetter, K. Riemenschneider, S.D. (1983) Birkhoff Interpolation, Addison-Wesley Publishing.
[9] Neumann, E. (1981) cubic spline with given values of the second derivatives at the knots, Demonstratio Mathematica XIV, No. 1, 155-125.

[10] Saxena, A. , Singh Kulbhushan; Lacunary Interpolation by Quintic splines, Vol.66 No.1-4 (1999), 0-00, Journal of Indian Mathematical Society, Vadodara, India.

[11] Schoenberg, I.J. (1972) Notes on spline functions, I, Koninkl. Nederl. Akademievan WestenschappenAmesterdam, Series A 75, No. 5 and Indag. Math. 34 No. 5. 